\title{
Filigrane
}

Écoutes psychothérapiques

\section{Quand la mer se retire suivi de Les lieux-dits du rêve}

\section{Brigitte Bournival et Nane Couzier}

Volume 16, numéro 1, printemps 2007

Les hauts lieux et non-lieux du rêve I

URI : https://id.erudit.org/iderudit/016177ar

DOI : https://doi.org/10.7202/016177ar

Aller au sommaire du numéro

Éditeur(s)

Revue Santé mentale au Québec

ISSN

1192-1412 (imprimé)

1911-4656 (numérique)

Découvrir la revue

Citer cet article

Bournival, B. \& Couzier, N. (2007). Quand la mer se retire suivi de Les lieux-dits du rêve. Filigrane, 16(1), 61-79. https://doi.org/10.7202/016177ar
Résumé de l'article

LES LIEUX-DITS DU RÊVE : BRIGITTE BOURNIVAL

Une question est née entre deux femmes, l'une poète, l'autre psychanalyste : "Le Lieu à partir duquel le poète aspire à nommer le Monde, ne serait-il pas le Lieu d'où le psychanalyste tente d'entendre le Sujet dans son énonciation ? " Dans son mouvement même, cette interrogation s'offrait à l'évidence d'une parenté certaine du Dire poétique et de l'Entendre psychanalytique avec l'espace du Rêve.

La femme psychanalyste choisit aujourd'hui de laisser au poète la parole, parole qui permet à la doublure nocturne du Vivant de se revêtir du Grand Jour.

QUAND LA MER SE RETIRE : NANE COUZIER

Cette suite poétique propose un retour à la mer disparue - une avancée dans la dispersion des âges, des signes, des temps.

L'espace d'écriture, que sillonne une quête des commencements de soi, adhère à des étendues successives où regard et voix progressent en même temps que bougent les paysages traversés.

Au bout de cette " narration cachée " : le lieu primordial d'un Ailleurs à la langue de sable, aussi vaste que le Temps. 


\section{Quand la mer se retire suivi de Les lieux-dits du rêve}

\section{brigitte bournival et nane couzier}

LES LIEUX-DITS DU RÊVE : BRIGITTE BOURNIVAL

Une question est née entre deux femmes, l'une poète, l'autre psychanalyste : «Le Lieu à partir duquel le poète aspire à nommer le Monde, ne serait-il pas le Lieu d'où le psychanalyste tente d'entendre le Sujet dans son énonciation?»

Dans son mouvement même, cette interrogation s'offrait à l'évidence d'une parenté certaine du Dire poétique et de l'Entendre psychanalytique avec l'espace du Rêve. La femme psychanalyste choisit aujourd'hui de laisser au poète la parole, parole qui permet à la doublure nocturne du Vivant de se revêtir du Grand Jour.

QUAND LA MER SE RETIRE : NANE COUZIER

Cette suite poétique propose un retour à la mer disparue - une avancée dans la dispersion des âges, des signes, des temps.

L'espace d'écriture, que sillonne une quête des commencements de soi, adhère à des étendues successives où regard et voix progressent en même temps que bougent les paysages traversés.

Au bout de cette «narration cachée» : le lieu primordial d'un Ailleurs à la langue de sable, aussi vaste que le Temps.

nane couzier

\section{Quand la mer se retire}

Qui peut dire où commence la marche.

Si vraiment elle a commencé.

Depuis quand.

\section{La marche commence-t-elle dans le cri?}

Il fait noir

plus noir que d'habitude

la ville répand ses lumières livides.

Un bruit de fond racle l'espace.

Entre des murs posés quelque part sur l'asphalte

un cœur bat

quelqu'un attend. 
Un cri longuement crève le manque d'air se jette dans le vide.

On ne sait d'où vient ce cri ni pourquoi on le pousse au-dehors tout à coup.

Au passage il fracture le cœur qui attend là.

Le cœur s'ouvre un souffle se perd.

Le cri emporte les murs les chambranles remue tout.

Rien n'est à l'abri.

Dans la bousculade

le noir s'effondre sur les rares lueurs encore bleues de la nuit.

Le cri rejoint les voix inanimées

l'air disparu

les lieux déjà engloutis par le temps.

\section{La marche commence-t-elle dans l'appel?}

Longtemps j'ai habité la mer son souffle couvrait les rivages en elle tout respirait.

Un jour elle m'a laissée la mer.

Un temps j'ai cru à son retour il n'y a pas eu de retour.

Elle est partie sans hâte sans rupture dans un surcroît de clarté. 
Le ciel ouvert

de la lumière s'est abattue

sur elle

des poches ont pris corps depuis le fond

des creux

si noirs

masqués jusque là.

Aspirée par le noir

elle est partie, la mer.

J'ai attendu.

Un désert s'est avancé sur la plage

on entendait craquer la surface

le sel

déjà sec.

Le désert menait très loin où les paysages se touchent

là-bas

le soleil dans les plis de la mer

j’ai suivi le désert.

Des silhouettes blanches m'ont accompagnée

spectres de poissons morts mêlés à l'odeur criante des vases

à la soif des palétuviers sur les berges

le jour a décliné rapidement devant la procession

des restes marins.

Le désert s'est arrêté au pied d'une formidable chute

rouge

des touristes passaient au-dessus

un à un

des poissons lune tombaient de la chute

avalanche de cercles blancs

le lit d'oursins en bas

où s'empalaient leurs ventres laiteux

j’ai regardé ailleurs. 


\section{4}

Filigrane, printemps 2007

Et j'ai vu les derniers atolls de lumière s'éteindre

deux mains indifférentes ont dispersé lentement les décombres quelque chose a bougé sous le sable

je me suis approchée pour voir.

Il se mit à faire bleu des vaguelettes se pressaient sous la lune muettes

leur présence malléable émergeant du plus sec de la mer.

Un bruit d'avion quelque part dans le bleu me fit songer nous avions survolions les mêmes paysages ... toi et moi...

ne faisions qu'un qu'une ... mer et eau... ... sable et dune...

un tremblement des vibrations le choc

nous avons dégagé nos ailes l'une de l'autre plané un moment

puis nous nous sommes tus.

Le bruit est repassé avec lui la vision d'une carlingue échouée deux gouffres enlisés dans l'épaisseur soyeuse du sable

sous le bleu

cet avion

perdu

naufrage parmi d'autres naufrages égarés dans le désœuvrement la quiétude les désastres oubliés. 
On voyait tout autour les côtes excavées de la mer ses bords luisants

son ventre vide

il faisait presque doux

l'impression de flotter

l'eau, le temps, le ciel, la nuit

confondus.

À la longue un nouveau jour me ramena d'où la mer était partie

le couchant me regardait depuis l'autre côté du vide

ses chutes rouges au loin

tout aussi assourdissant le silence

que les grondements

entre lui et moi

le départ des eaux

un espace encombré de lumière.

Ce nouveau jour finit par remplacer le bleu

la lumière se fit tranchante

l'air vibrant

j'aurais voulu retrouver le souffle de l'eau

les clapotis

les longues rames de conciliabules sur la grève

m'y bercer

plus rien ne respirait comme avant.

Les bruits de l'océan étaient perdus.

Du berceau vide j'ai rappelé la mer

et j'ai vu sa figure

cassée

tant de lassitudes

les cavités

son regard d'écaille

les traces de sel

brûlantes 
66

Filigrane, printemps 2007

je n'ai plus appelé.

\section{La marche commence-t-elle dans le rêve?}

Je suis devenue sans voix au départ de la mer

dans son absence

torride

c'est de là que j'écris

elle me manque

sa respiration surtout.

Il y a toujours cette masse rouge à l'horizon du côté du couchant

des milliers de bulles l'assaillent

la masse ne bouge pas

je sais qu'elle gronde

on ne l'entend pas d'ici

le grondement se perd dans la distance

une sorte de feulement me parvient.

Quelque chose semble pousser vers l'avant tout ce rouge

il penche démesurément

fait énormément d'ombre

il pourrait recouvrir tout le sable.

Les petites bulles se rassemblent peu à peu en son cœur.

En un blanc.

\section{La marche commence-t-elle dans l'exode?}

En d'autres temps aussi la mer s'est retirée un jour bien avant la naissance des hommes

avant l'arrivée du désert 
on le sait aux reliques trouvées dans les couches pétrifiées de l'océan le fond ouvert puis refermé sur l'âge de pierre.

Au plus profond de ce temps écoulé on retrouve les marques d'intimité de la mer disparue on en retrouve jusqu'au bout des déserts

c'est sur ce lot de temps que les hommes ont bâti leur histoire.

Les hauts plateaux du Tassili en plein Sahara ont gardé cette mémoire native des hommes

la pierre qu'ils ont habitée

les falaises rouges

les socles noirs

les colonnes fantomatiques

hauts fonds recreusés par les vents

sortis de l'érosion

usés sur toute leur étendue

après l'assèchement

avant que les longues artères enfoncées sous les corridors gréseux ne retiennent la pluie

l'eau douce.

Des siècles et des siècles de conversations muettes peuplent le sud du Hoggar des millénaires de silhouettes blanches dans le temps

fresques étalées par centaines sous la poussière d'argile

des corps à la volée sur les parois rocheuses

pour les voir il faut mouiller l'argile déposée au-dedans

raviver les ocres

laver la mémoire des grès.

Ces grottes racontent la prime enfance de l'homme ses débordements

le départ à dos de buffle

l'exubérance a cessé avec l'arrivée du désert

le tranchant des éboulis sous le sabot des bêtes

les longues traînées de sable croûté où se sont enfoncées les rivières 
le ventre chaud des grottes

devenu brûlant au-dessus des gueltas sèches.

Le paysage recèle encore de rares trésors sous les petits monticules de cendres abandonnés par-delà les oueds taris les chotts de sel

c'est ce que montre la pierre.

Par endroits le désert a gardé la marque du sol natal des hommes de grands cyprès s'accrochent encore à leur promontoire des pitons rocheux guillochés du dehors se souviennent de la légèreté des eaux de surface — quand la terre d'Afrique était verte.

\section{La marche commence-t-elle dans le doute?}

L'entrée dans la traversée du désert commence toujours de la même façon. Sans bruit.

Une respiration - un blanc: ce petit tapis propre au seuil des histoires neuves puis plus rien. L'invisible tremblement de l'air dans le temps.

De loin le désert semble épouser le vide. Le remplir de cet abandon où se trouve le sol

confiné à l'épuisement.

Invitation à la paresse - la mollesse des vagues de sable leur langueur le ralentissement des sens posés sur l'usure.

La poussière du désert se déplace dans la masse désertique. Les grains s'empilent, vont

ailleurs, progressent, gagnent d'autres terrains. Passent des frontières qu'ils engloutissent sans effort.

Rien ne bouge dans ce mouvement élémentaire — que le déplacement.

La lumière est partout

vide apparent où l'on se cogne par-delà les saillies

les échancrures du paysage. 
La lumière serrée sur elle-même. En apparence ouverte sans fard.

Fermée pourtant. Brutale. Compacte.

On reconnaît la marque du désert à cet indice de dureté absolue la lumière.

À cet étau transparent que brouillent de temps à autre les vibrations de l'air à l'annonce des mirages. Les nappes de chaleur sur le sol. Leur échappée vibrante.

\section{Comment}

sans une lente déperdition des sens survivre à l'absolu du désert. À sa dureté. À son emprise. À la constance des signes de dépérissement. Au désœuvrement qu'impose l'ordre sec à force de rien.

Comment.

Ce rien ultime et premier. Le bout de toute dépossession.

Ordre sans objet le désert consume. Jusqu'au ciel.

Il vaut mieux poser un regard flou sur cette brûlure. Le plus longtemps possible. Une vision défaillante. Pour empêcher l'aveuglement.

Quand l'éblouissement impose de faire halte il est trop tard.

L'entrée dans la traversée du désert commence toujours de la même façon — le petit tapis propre au seuil du dépaysement. Puis rien d'autre. Que le blanc. Trop blanc.

\section{La marche commence-t-elle dans la mort?}

Vous voici dans le blanc. L'entrée du désert. L'invisible.

Rien ne bouge.

Vous tentez de vous acclimater au silence compact des lieux après l'étourdissement.

La nouveauté des sensations vous occupe. Vous émerveille. Vous tendez l'oreille.

Votre ouïe se charge du vide ambiant. À la limite du supportable. Le manque de mouvement vous saisit. Vous cherchez à voir. N'importe quoi. 
Rapidement, vous distinguez du paysage ce qu'en disent les lieux communs — les fossettes d'ombre au creux des dunes ; leurs crêtes bien peignées ; les plissements, les replis, les froissements silencieux du sable. L'image vous rassure.

Vous ne saisissez pas encore la netteté infernale de cette perception. Vous suivez des yeux. Longuement. Sans véritable regard sur ce qui s'affiche.

$\mathrm{Au}$ bout d'un petit ennui, un brin d'agacement. Vous tentez de faire bouger quelque chose: une fillette surgirait, suivie de ses chèvres...

Vous hâtez le mouvement; pressez la scène d'avancer; ordonnez à l'image: un cavalier ferait irruption, enlèverait la fillette et dans un nuage de poussière, échapperait à ses poursuivants...

Une seconde, vous croyez avoir imposé au désert votre vision du temps. Puis vous entrevoyez l'effort qu'il vous faudra faire pour maintenir l'artifice. Votre volonté soudain

se rompt. L'image, désenchantée, se ferme. Un regard plus grand que le vôtre vous siphonne. Le paysage se met à tourner. Votre œil cherche d'urgence à rétablir son cadre. Une pellicule grise masque du dehors votre vision en même temps qu'un objet dur en griffe la surface. La troue.

Vos yeux ont mal.

Vous entrez dans l'opacité réelle de votre vision.

Dans la matière même de l'œil.

Dans sa substance.

Votre œil alors grossit l'alêne qui le perce. L'objet sur lequel vous avez buté : un cadavre décharné de bête — chèvre, cheval, dromadaire... sans différence. Le reste arrive très vite.

Vous entrez dans la bête. Au plus profond.

Vous voilà maintenant l'œil dans l'œil désaffecté de la bête. Vous hurlez. Le cri résonne très loin. Vous l'entendez se perdre. Les parois du crâne étranger contre le vôtre. Votre œil dans son œil troué. Vous, pressée de toutes parts. Broyée vive.

Il faut pousser. Expulser l'étau qui se resserre. Ce masque sur votre visage. La pression des os.

Un nuage rouge puis le crâne vous éjecte sans transition dans l'air brûlant. Le contact abrasif avec le sable — à sec. Sa pénétration. Votre peau, votre tête raclées du dedans.

Les paupières, les narines embrasées. Vous, écrasée par l'arrachement. Jetée dans le tranchant de l'air. Son incandescence. 
La poudre salée autour de la bouche, déjà — l'écume sèche de la soif. Ce relent saumâtre de vie morte en vous.

Choisir de vivre. Marcher. Avec ce corps brûlé. Pesant. Avec la soif qui fera oublier l'insalubrité des eaux de surface. Doucement se mouvoir dans ce mal de tous les instants. Se contenter de n'en rien savoir. Avancer un bras, une jambe. Dormir. Oublier. S'habituer au désenchantement: l'économie des gestes, le brouillage perpétuel des repères sur le sable, l'application à respirer de tous les instants. Le ciel à la limite constante du déchirement.

Tout ce peu, il faut s'y habituer. Le désert ne mène nulle part ailleurs. Que là. Dans les menus progrès du jour. Son visage si dur qu'on a peine à se souvenir d'autre chose.

Cette rencontre - la dureté même - c'est à la fois le bout du désert et son renversement. La permanence établie du rien. L'avant et l'après confondus de toute chose.

La terreur passée vous déposerez vos restes diurnes quelque part dans le temps. Des restes que personne n'aura entendu venir.

Ces restes affleureront des soubassements du monde. Votre épave de lumière flottera dans la fournaise. L'embrasement. Avec celles des bêtes. Et les roses de sable.

La vision renversée vous irez dans le plus clair du temps.

\section{La marche commence-t-elle dans le temps?}

Vous voilà dans la nuit.

Devant vous, la lune. Sa présence. Vous pourriez y toucher.

Quelqu'un marche. Vous marchez dans son pas.

L'amplitude de ce pas vous oblige à de grandes enjambées.

Dans la foulée vous n'entendez pas que vous vous rapprochez de l'être qui vous précède.

Sa marche est lente. Vous dans ses traces, plus rapide.

Vous sentez sous vos pieds l'empreinte - le sable embossé. Le sol habité avant vous par cet autre. La sensation du sable sous le pied vous distrait. Vous avancez le souffle haut. Vous ne savez pas encore marcher dans la nuit. Dans le temps lâche de la nuit. Dans sa respiration. Sa clarté. 
Un corps immense soudain vous arrête. Vous attend.

Le bruit d'avion glisse sur le silence. Vous le suivez des yeux sans le voir.

L'autre s'adresse à vous. Sa voix résonne. Il parle dans une langue très ancienne. Native des lieux dirait-on. Vous entendez les sonorités de cette voix.

Entre les sons égrenés par cette voix il y a le drapé de la nuit. Et vous dedans. Au fond du monde. Au plus intime. La chair du temps.

Celui qui vous précède se remet en marche. Lentement. Vous ralentissez, le laissez maître des lieux en ménageant un peu de distance.

Il arrive au faîte d'une colline. Sa robe un peu plus sombre que l'indigo de la nuit. Son geste vous signale quelque chose. Vous écoutez.

Le bruit de la mer. Vous la respirez presque. Elle est là. Qui souffle, râle, chuinte, grésille. Se meut.

Le plaisir est immense. Intenable. Vous suffoquez.

L'homme de la nuit attend un peu puis vous indique une direction. Il vous fait signe de suivre le rivage.

Vous empruntez ce chemin et longez le glissant de l'eau. Vos traces dans la grève humide près de celles des petites bêtes venues attendre la rosée du matin.

Vous vous retournez une première fois. L'homme de la nuit est là. Sa robe flotte.

Vous vous retournerez de nouveau. Il aura disparu.

Vous porterez la marque de sa présence. Son étoffe plus dense que le bleu de la nuit. Détachée du ciel.

En vous.

Peut-être lui donnerez-vous un nom.

Peut-être pas.

La marche commence t-elle dans l'oubli? 
brigitte bournival

\section{Les lieux-dits du rêve}

Voilà donc cet espace infini qui s'ouvre devant nous...

Une question est née entre deux femmes, l'une poète, l'autre psychanalyste: «Le Lieu à partir duquel le poète aspire à nommer le Monde, ne serait-il pas le Lieu d'où le psychanalyste tente d'entendre le Sujet dans son énonciation?»

Dans son mouvement même, cette interrogation s'offrait à l'évidence d'une parenté certaine du Dire poétique et de l'Entendre psychanalytique avec l'espace du Rêve.

C'est depuis ce lieu tendu entre deux extrêmes, lieu de l'impossible, près du Rien, qu'elles se sont écrit pour entendre d'où elles parlent, pour se dire d'où elles entendent.

Parcourant leur rencontre définie par la fonction même de leur rapport à la Parole, elles espéraient réduire la dette de Chair qu'elles savaient imputable au Mot.

Mais se demandaient-elles, le lieu du dire poïétique, l'antre de «l'attention flottante », la grotte du rêve sont-ils espaces d'un même Lieu?

Que se passe-t-il lorsque l'endormie ne répond plus au présent du courant de la vie et plonge les racines de son être dans le mouvement paradoxal du sommeil?

Quelle découverte est en survenance quand le poète en creusant la Langue pour la faire parler, entame et ainsi fait que s'entend la Mémoire du monde?

Qu'advient-il lorsque le psychanalyste ose entendre la voix de la langue première, le cri du mot dans la parole?

Au début de l'aventure, la psychanalyste s'adressait ainsi au Poète :

Poète, fais pour moi effet de l'Un

Parlons-nous à Deux

Désignons par nos deux voix

Les deux bords de la Marmite Infernale

Qui composent le Réel

Tâchons d'entendre le «NOUS » qui traverse la langue

Ainsi notre cri ne sera plus béant de bouches ni muet de sons

Il appellera la clameur du Rêve

Le Rêve se tiendra alors Là

Devant nous, entre nous, autour de nous 
Nous n'aurons le choix que de nous y soumettre

Pour ne pas s'y noyer

Ensemble nous tenterons de dire

Dire au plus près de la bouche du mot

Pour entendre la voix évocatoire d'une Première Mémoire

Se mettre à la table de la Parole

Sera donc s'offrir à la substance du Rêve

Subir la fougue de son mouvement

Faire face à l'effroi de son tumulte

Brûler de la beauté de ses lumières

Porter sa parole vers l'Autre

Fera alors parler le Rêve

Ainsi Fleuve il deviendrait

Et nos voix, reprenant l'écho de ses Rives

Chanteraient ses «chants de gorge»

Scandant le rythme du «Chemin qui marche»

Ici l'Origine se ferait Souffle

Trace au plus près du creux de la Langue

Nous assurant de notre avancée

Vers des terres encore plus lointaines

Poètes et psychanalystes, êtres de corps, de sang et d'étendue d'âme, nous sommes «des échourés ».

Nous sommes les échouements du Rêve, les échoués d'un rêve (celui d'un père-mère), les «dé-noyés» d'une Première capture maternelle qui avait le pouvoir d'être

Mortelle.

Poète ou psychanalyste nous savons que le fait même d'échouer nous offre à l'ex-sistence, que c'est grâce à une dé-portation hors du sein des eaux que nous avons été appelés à naître.

Poète-psychanalyste, nous nous offrons à la certitude que c'est par le creux du mot que non seulement nous parlons mais que nous pouvons entendre la vague du fond de l'être.

La femme poète et la femme psychanalyste, tout au cours de leur conversation écrite, ont tenté de se garder dans le Lieu de l'impossible, près du Rien, attentives au leurre des formes du Rêve, lisant ses déguisements, mais se réjouissant pourtant de ce qui parvient à s'énoncer au cœur du Rêve, dans ses topographies, dans l'éloquence de ses représentants, dans la présence constante de la préséance des siècles. 
Toutes deux entendent et disent combien, par ses lieux-dits si précis, ses paysages hors saison, ses foules, sa démesure, son humour voyou, ses scènes macabres, l'espace du Rêve nous rend au Lieu tragique du DÉSIR NON ENCORE DÉ-VOILÉ.

Ainsi, pour chacune d'entre elles, il apparaissait que le lieu d'où elle parle, le lieu d'où elle entend a partie nouée avec le Rêve, MÉMOIRE DU MONDE, MOUVEMENT DE LA TRACE, HABITACLE DU DÉSIR.

\section{Le lieu du rien, toile de fond du rêve}

Quel est donc ce lieu du dire poïétique et de l'entendre psychanalytique?

Lieu dévêtu du «moi » et inscrit au-delà du «Je», ère du primordial et de l'ultime, vastitude du nom caché qui offre une trace à l'origine. Ce lieu, aux confins de la voix marque autant un espace de mise-au-monde de la parole que de sa mise-à-mort. Il est au carrefour de l'indiscible et de l'indécidable, possible «lieu-entre-deux-morts », celle déjà advenue par voie de Naître et qui appelle l'autre à venir par voie d'un mourir.

C'est un lieu accordé aux rives du silence, de «La Chose» tue, source des larmes d'où on entend encore la sonorité des eaux en dormance.

C'est un lieu vide, immensément vide et pourtant porteur du nœud du monde qui se creuse pour porter en son sein l'enracinement pour toujours de «l'ombilic du rêve».

Le lieu du Rien, s'offre au poète, au psychanalyste mais aussi à l'Enfant comme caisse de résonance des signifiants-vocables que la Mère aura gravé dans leur chair, les rattachant ainsi à la chaîne des mémoires ancestrales.

Ce lieu, toile de fond du Rêve est le lieu même de la Chute, de la déchirure de la Peau du Monde qui, un jour de naissance a offert l'enfant à la lumière de la Mémoire.

\section{Lieu de la chute, bornage du lieu du rien}

Le lieu du Rien est un lieu impossible à nommer, sinon à se faire les témoins d'un «perdu à jamais». Ce lieu nous raconte non seulement notre Chute pour naître mais l'Exil qui s'est imposé à nous bien avant notre Exode, ce commencement de notre mise-en-marche.

Mais encore, comment dire ce lieu si proche de l'absence des mots?

La psychanalyste dira:

«Nous n'avons que nos rêves pour dire le lieu d'où l'on vient, pour entendre la source du chagrin qui nous fonde, pour sortir de l'oubli. Nous ne portons que nos bégaiements de langue (lapsus) pour approcher cet espace d'où nous avons été parlés. Nous ne possédons que notre humour (mot d'esprit) pour nous révéler dans la boiterie de notre marche. » 
Mais la certitude que la parole se fonde sur ce manque fait office pour le psychanalyste de lieu-dit où elle se tiendra pour entendre car elle sait que nous devons à la cruauté du manque le tissage même des fils du désir.

\section{SON SAVOIR FERA D’ELLE UNE «DÉS-ENDORMIE» QUI APPELLE LE RÊVE POUR CESSER D'OUBLIER..}

La femme poète se tiendra en ce Lieu même du Rien, pour entendre la Parole qui lui parle au-delà de son «Je». Son écriture adviendra de ce Lieu mais au prix chaque fois d'une précipitation au cœur de la noyade, de déchirures oubliées surgissant du sein de sa langue et de longues stases qui appelleront le mouvement de la trace avant même que le Récit ne puisse s'amorcer pour dire la Mémoire.

\section{SON OFFRANDE FERA D'ELLE UNE PYTHIE AU CORPS SACRIFIÉ.}

L'Enfant, elle, sait «la lettre», le fin mot de «La Chose» tue et la parlera avant de pouvoir l'oublier.

Sur le seuil de la fin d'une séance, une enfant me demande du haut de ses 6 ans :

— Pourquoi y a toujours des semaines prochaines?

- Je ne sais pas, lui ai-je répondu, peut-être parce que nous avons encore des choses à nous dire mais je pense savoir que c'est surtout parce que nous sommes vivantes, toi et moi.

— NON, ME RÉTORQUE-T-ELLE, DES SEMAINES PROCHAINES C'EST QU'ON VA MOURIR... ET ÇA J'EN VEUX PAS !

C'est ainsi que l'Enfant me parlait de la brûlure du temps mais surtout de sa découverte tragique que le Temps nous offre à la Mort. Je ne pouvais éprouver moi-même ce sentiment du tragique qu'elle me révélait par ses paroles qu'à la condition de me savoir déjà effet de ma propre mort.

C'est de ce Lieu même où je n'espérais ni la torturer, ni la consoler, simplement lui indiquer que Là était l'autre «premier pas » à faire pour continuer de vivre.

À la suite du vertige enfin réalisé, l'enfant me dira :

- Racontons quelque chose qui ne se passe pas du tout dans la vraie vie... quelque chose de l'ancien temps. 
Elle s'empressera alors de situer son récit dans la ville où elle était née. Oui, ce que nous avons perdu, ce «perdu à jamais » qui nous répercute dans «la vraie vie » se présente à nous comme sans cesse à re-perdre pour se découvrir EN DÉSIR, accordés à «l'ancien temps ».

SA DÉCOUVERTE DU TEMPS QUI FAIT MOURIR FERA DE L'ENFANT UN SUJET QUI S'OFFRE AU RÉCIT, S'ASSURANT AINSI D'UN ACHEMINEMENT VERS SA PAROLE.

\section{MAIS QU'ADVIENT-IL DES TROIS OCCUPANTES DE L'EXIL?}

Occupantes du Lieu du Perdu, Toile de fond du Rêve, lieu de l'Exil avant l'Exode, chacune d'Elles dira, saura et adviendra à sa façon particulière et multiple.

La femme poète n'a pas le choix d'y être et de rester seule en ce Lieu d'Exil, car c'est seulement à partir de Là que lui est donné la Parole inspirée. C'est ainsi qu'elle parlera au-delà du «JE». C'est ainsi qu'elle se fera témoin unique d'un Lieu qui lui souffle sa voix à l'oreille.

\section{LA FEMME POÈTE, IMMOLÉE PAR LES FEUX DU DÉSASTRE ÉCRIRA POUR NE PAS MOURIR.}

L'Enfant, elle, est catapultée en ce Lieu, pour advenir. Mais elle ne veut pas y être.

Son refus de la Mort permettra au Sexuel de s'arrimer, faisant d'elle une enfant, mûe puissamment par des pulsions à vivre.

\section{L'ENFANT DIRA À LA LETTRE LE LIEU DE L'EXIL MAIS SANS SAVOIR QU'ELLE L'ÉNONCE. ELLE DIRA POUR DEVENIR.}

La femme psychanalyste choisira de s'approcher du Lieu du Perdu pour entendre ce que l'Enfant a à lui dire. Mais à la différence du poète, elle ne tiendra pas Seule en ce Lieu. Pour y être, une exposition-à-deux lui sera nécessaire.

Elle s'offrira donc à la demande de l'enfant, pour représenter une présence au-delà d'elle-même, présence qui n'oubliera pas l'absence qui la fonde. Elle deviendra «objet d'amour ou de haine», sans pourtant s'y croire afin que «l'objet perdu à jamais. » re-prenne figuration sous un de ses traits et que l'enfant puisse s'approcher à nouveau de l'objet dans sa Chute, de la nomination de sa perte et ainsi pouvoir advenir à sa mémoire par l'oubli mais pas sans le retour, du tremblement de terre, des déflagrations nécessaires à la reprise, à la résurgence des premières chutes. 
C'est ainsi que le Transfert, nécessaire à l'acte analytique ouvrira une voie dans le parcours de remémoration de l'enfant, tracera la ligne d'un mouvement pulsionnel et parfois permettra l'énonciation d'un véritable récit.

La femme psychanalyste s'exposera au Transfert pour en savoir un brin de plus sur l'Amour tout en favorisant pour l'enfant, le pas suivant qui est nécessaire à la mise-en-marche de son Désir.

LA FEMME PSYCHANALYSTE ENTENDRA LE RÉCIT POUR FAIRE SAVOIR QUE «... L'AMOUR RÉALISÉ DU DÉSIR DEMEURÉ DÉSIR » (Char, Sur la poésie, p. 10) EST POÈME MAIS AUSSI RÊVE DU DÉSIR ET ACTE ANALYTIQUE QUI S'Y FONDE.

Ainsi, nous pouvons énoncer que les trois Occupantes du lieu du Rien, lieu du Perdu, lieu de l'Exil, LIEU-DIT DU RÊVE ont choisi, par décision d'entendre, par balbutiements de vivre et par nécessité d'être, DE VIVRE LA MORT COMME ESPACE DE COMMENCEMENT.

C'est pourquoi leurs voix se fondent et entonnent la louange de la déclivité, lieu même de leur abondance. AINSI LE RÊVE SERA APPROCHÉ COMME BOUCHE DU RÉEL ET PLACENTA DE LEURS MOTS.

«C'est ici, que parvenue au silence de l'énigme, je rencontrerai peut-être l'homme au manteau de nuit et que son chapeau ne partira pas au vent même si j'ose suivre les traces de son pas jusqu'à la Mer et que Seule ensuite, je saurai m'y tenir sans affolement ni prétention, comme il se doit, pour continuer d'entendre... la réalité des êtres qui me parlent de la mer qui se retire et qui parfois découvrent dans l'entre-deux de la scène... l'Idéal qui porte au pas suivant en s'offrant à la force du Souffle qui Lui, continuera toujours d'aller où Il veut. »

C'est à partir de cet espace de déréliction et de réalisation que la femme psychanalyste choisit aujourd'hui de laisser au poète la parole, parole qui permet à la doublure nocturne du Vivant de se revêtir du Grand Jour.

Ici, au Lieu du Poète, aucun éloge du Rien, juste le courage désespéré d'y être, d'y rester pour que le «muet» de la langue s'extirpe de la Nuit, dessinant ainsi l'espace du Dire.

Ici, je me fais «oreille», attention flottante à l'écriture du poète, car sa langue même vient donner corps aux états premiers du Sujet.

Un Sujet n'est-il pas à naître dans un espace-entre, creux entre-deux-êtres, lui-même sculpté, évidé par le trait d'un troisième, qui par sa présence offre au regard une visée et au pas sa portée. 
Car où rencontrer un Sujet, une âme humaine, sinon dans l'espace-entredeux coupures, l' Une qui s'évoque par la disparition du Tout de la mère, l'Autre qui apparaît par l'Idéal offert au pas suivant?

N'est-ce pas entre mémoire, mouvement et récit, les premiers états du Rêve qu'un sujet naît et émerge pour advenir?

La femme poète dans son écrit «Quand la mer se retire», nous annonce que si le Rêve est Origine, la coupure, scandée d'une double disparition est Commencement. C'est dans l'infime amorce du commencement que le Sujet advient et que le Rêve continuera de tendre l'oreille à la réalité des mots.

brigitte bournival 910 , rue querbes outremont qc h2v $3 \times 3$

b.bournival@qc.aira.com

nane couzier 690 , chemin saint-antoine les cèdres (québec) $\mathrm{j} 7 \mathrm{t} 1 \mathrm{~g} 3$ nane.couzier@sympatico.ca 